\title{
nature
}

\section{Challenges of the Grid}

Science policymakers worldwide are waking up to the opportunities of 'the Grid' - a supercomputing network transforming many disciplines. But it poses new organizational challenges for researchers and their institutions.

A fter the ILOVEYOU virus outbreak, a cartoon in the newspaper Le Monde portrayed a company boss thanking his assembled staff profusely for being so computer illiterate as to be still ignorant of how to open an e-mail attachment. Thankfully, the computing savoir faire of the average scientist is considerably greater. Yet there is an irony. The desire of scientists to collaborate inspired the invention of the World-Wide Web at CERN. But whereas the Internet has since infiltrated every inch of the planet, its use for widespread scientific collaboration - apart from a web-wise minority - remains largely rudimentary compared with the potential offered.

For most scientists, the web remains a place to search for information. For many, building a laboratory home page is the closest they come to interacting with the medium, and most of these sites are static; the electronic medium may mean that they are immediately accessible to all, but they provide users with little interactivity. Highly interactive laboratory pages, where, for example, any member of a community can edit databases and pages online, remain the exception.

One reason can be found in the dominance of proprietary software. Microsoft's 2000 release of its Office suite, for example, brings collaborative web working tools to such workhorses as Word and Excel. But in order to benefit, every user must have Office 2000 and Microsoft server extensions. Barring a considerable investment in new software, this makes a collaborative web environment difficult even in an intranet where everyone is using Microsoft products and PCs. In the Internet environment, where users may be using Macs, Linux or Unix operating systems, and a multitude of programs, such closed systems are not a solution.

\section{Open and free}

As it happens, simple open-source and highly interoperable tools for collective working exist, and are often superior to their commercial counterparts. Apart from the costs of hardware, it is possible to build an excellent site, for example, using the Linux operating system, an Apache WWW server and the MySQL database. To this one can add such features as WikiWeb, a collaborative website that can edited by anyone (http://www.ensembl.org/Docs/wiki/html/EnsemblDocs/ GettingStartedWithWiki.html), a simple web database interface (http://www.ofifc.org/Eli/ASP/GenericArticle.asp) or a means for users to upload any sort of file online (http://www.dougdean. com/EZsiteUpLoadSite.htm) — all are free, with most requiring only a minimal knowledge of computing and programming languages.

Plans that will encourage a more sophisticated and highly productive use of networks are now afoot. The National Computational Science Alliance in the United States and, more belatedly, the European Union, are enthusiastically embracing an ambitious architecture for the next-generation Internet: a 'computational Grid'. The concept is elegant. Few of us have the benefit of a supercomputer next door. In the Grid, any scientist could plug their computer into a high-speed Internet and immediately share huge supercomputing resources and in particular supercomputer 'clusters' of cheap PCs - distributed across a high-speed Internet. The increasing occurrence of tera- and petabyte/flop datasets, data flows and computations in science is creating a real demand for such computing power in many disciplines. But perhaps the most significant vision is one of an Internet where all sorts of datasets, visualization tools and software can communicate in the same language. This could be achieved through a software layer - with the uninspiring name of 'middleware' - that would allow anyone on their desktop to draw on datasets wherever they were held on the Internet without having to worry about formats or protocols. The Internet becomes the computer.

\section{Collaboratories}

At a recent 'E-science' workshop hosted by the European Commission, the backing for grids from disciplines as diverse as genomics, particle physics, space science and meteorology was, to say the least, wildly enthusiastic. The vision is well-founded. In short, it is one where people, computers and services no longer operate in isolation, but are shared and made available to the entire community; individual laboratories would give way to international 'collaboratories' where geographically dispersed groups would work seamlessly on shared datasets; it is already happening (see Nature 402, C67-C70; 2000).

Plans for grids are dominated by the high-performance supercomputing community, mainly physicists. Their leadership promises benefits to other less computing-savvy areas of science. The immediate next steps in the Grid projects are eminently laudable, for example to build high-speed networks, impose order on disorder by allowing databases and other applications to speak the same language.

But there is a danger in such top-down approaches, namely that the technology risks becoming an end in itself, removed from the real needs of diverse grassroots users. In the early days of physics, and more recently in genomics, the major strides - such as Ensembl (see page 333) - have largely been driven by a 'hacker culture' where computerliterate scientists designed and built information-technology systems to satisfy urgent and pressing bottom-up research needs. The challenge will be to merge these two cultures.

Using current browsers, anyone can access dumb HTML documents. Grid browsers will allow scientists just as easily to access computing resources, databases and tools. Using XML, CORBA and other languages and protocols, it is already possible for websites and online databases to communicate, interpret and understand one another's content. It is up to individual communities to organize themselves to take advantage of this opportunity.

The Grid concept is without doubt the way to go, and will benefit scientists greatly. But to maximize the benefits of the next-generation Internet in an optimal, sustainable and rapid way, it cannot be left to either the enlightened vision of a few computing gurus or the heroic effort of entrepreneurial scientists at the bench concerned with real research problems, doubling up as computer scientists.

It is a common, and perhaps unjust, maxim that whenever you want to do something progressive in computing, the last place to look is your institution's IT department. Nevertheless, one way or another, all institutions involved in science need to give priority to how modern information technology can best serve them, and equip themselves with the necessary budgets and competence. 